\title{
Development of Frequency-Fixed All-Pass Filter based Single-Phase Phase-Locked Loop
}

Gautam, Samir; Xiao, Weidong ; Lu, Dylan Dah-Chuan; Ahmed, Hafiz; Guerrero, Joseph M.

\section{IEEE Journal of Emerging and Selected Topics in Power Electronics}

DOI:

10.1109/JESTPE.2021.3085124

Published: 31/05/2021

Peer reviewed version

Cyswllt i'r cyhoeddiad / Link to publication

Dyfyniad o'r fersiwn a gyhoeddwyd / Citation for published version (APA):

Gautam, S., Xiao, W., Lu, D. D-C., Ahmed, H., \& Guerrero, J. M. (2021). Development of Frequency-Fixed All-Pass Filter based Single-Phase Phase-Locked Loop. IEEE Journal of Emerging and Selected Topics in Power Electronics .

https://doi.org/10.1109/JESTPE.2021.3085124

Hawliau Cyffredinol / General rights

Copyright and moral rights for the publications made accessible in the public portal are retained by the authors and/or other copyright owners and it is a condition of accessing publications that users recognise and abide by the legal requirements associated with these rights.

- Users may download and print one copy of any publication from the public portal for the purpose of private study or research.

- You may not further distribute the material or use it for any profit-making activity or commercial gain

- You may freely distribute the URL identifying the publication in the public portal ?

Take down policy

If you believe that this document breaches copyright please contact us providing details, and we will remove access to the work immediately and investigate your claim. 


\title{
Development of Frequency-Fixed All-Pass Filter based Single-Phase Phase-Locked Loop
}

\author{
Samir Gautam, Student Member, IEEE, Weidong Xiao, Senior Member, IEEE, Dylan Dah-Chuan Lu, Senior \\ Member, IEEE, Hafiz Ahmed, Senior Member, IEEE, and Joseph M. Guerrero, Fellow, IEEE
}

\begin{abstract}
Phase-locked loops (PLL) are widely used in the synchronization of grid interfaced power converters. One solution is based on orthogonal signal generation (OSG), which requires the grid frequency information for their appropriate operation.This paper developed a new solution to achieve the PLL function for single-phase grid interconnection but eradicate additional frequency feedback loops in the traditional architecture of all-pass filter PLL (APF-PLL). Four new topologies are developed along with their small-signal modeling and dynamic analysis. A thorough comparison among them on their dynamic response, steady state accuracy, implementation, and disturbance rejection capability are carried out. Finally, the best approach of frequency-fixed APF-PLL is experimentally evaluated with frequency adaptive APF-PLL and frequency-fixed PLLs belonging to time-delay (TD), and second-order generalized intergrator (SOGI) families.
\end{abstract}

Index Terms-Grid-synchronization, phase-locked loop, allPass Filter, frequency-fixed orthogonal signal generation

\section{INTRODUCTION}

$\mathbf{S}$ YNCHRONIZATION unit is a key element in grid interfaced converter (GIC) control systems [1]-[3]. Gridsynchronization based on phase-locked loop has been widely utilized to track the grid phase, frequency, and amplitude in single-phase systems. The PLL is a closed-loop control system constituting three parts, namely: phase detector (PD), loop filter (LF), and voltage-controlled oscillator (VCO) [4]. In general, all single-phase PLLs can be classified into either as (i) power-based or (ii) quadrature based PLL, depending on how the PD is implemented. The latter technique using OSG unit is primarily preferred for its distinct advantages, specifically, the absence of second harmonic oscillation and simplified amplitude estimation [5]. The objective is to generate a quadrature signal with the same magnitude as the fundamental component (grid voltage) for applying the synchronous reference frame (SRF) transformation. A wide variety of OSGs have been recommended for PLL application in the literature, such as TD [6], Hilbert transform (HT) [7], discrete fourier transform (DFT) [8], SOGI [9], all-pass filter (APF) [10] and complex coefficient filter (CCF) [11].

S. Gautam and W. Xiao are with the school of Electrical and Information Engineering, The University of Sydney, Australia (email:samir.gautam@sydney.edu.au; wxiao@iee.org

D. Lu is with the School of Electrical and Data Engineering, The University of Technology Sydney, Sydney, Australia (e-mail:Dylan.Lu@uts.edu.au)

$\mathrm{H}$. Ahmed is with the Nuclear Futures Institute, Bangor University, Bangor, LL57 1UT, United Kingdom. (e-mail:Hafiz.h.ahmed@ieee.org)

J. M. Guerrero is with the Department of Energy Technology, Aalborg University, Denmark.(e-mail:joz@et.aau.dk)
The APF presents a straightforward solution yielding an unity gain for all frequencies, while providing $90^{\circ}$ phase shift between the input and output signals at a specific (tuned) frequency. The research on APF-PLL has focused mainly on improving its disturbance rejection capability [12]-[14], discretization approach impact [15], governing dynamic speed [16] and small-signal modeling [10], [13]. The APF is adapted to grid frequency variation via an additional frequency feedback loop. This adjustment preserves the orthogonality of the output signal to the grid voltage, but creates an interdependent loop, difficulties in linearization, variable transfer function, and an intricate control problem [17]. To decouple OSG units from the PLL control loop, the concept of nonadaptive OSG has been introduced, where the OSG parameters are held fixed and ensuing errors are negated using separate structure. The additional compensator network can be designed either using an analytical or numerical method. As each OSG has different characteristics to generate an orthogonal signal set, each warrants a distinct frequency fixed configuration and requires separate design and analysis. Such frequency-fixed (FF) OSG filter has been applied to TD-PLL [6], [18], [19], SOGI-PLL [17], [20]-[22], CCF-PLL [11], [23], DFT-PLL [8], [24], and HT-PLL [25]. In contrast, only a few works are reported for frequency-fixed APF-PLL (FFAPF) but are found inadequate in meeting one or the other performance criteria.

Three different approaches for implementing the nonfrequency dependent APF-PLL have been compared in [26]. The two-stage FFAPF configuration shows satisfactory performance but only in limited frequency variation. Another two techniques, based on a compensator network and modified $d q$ transformation eliminated the phase offset error, while the second harmonic oscillation persisted. Furthermore, the smallsignal modelling was not developed and used for dynamic analysis. Another approach for the FFAPF-PLL employs an additional proportional resonant (PR) controller inside the control loop to eliminate the double frequency oscillations related to frequency variations [27]. However, it cannot eliminate the phase offset error and other details such as control parameters tuning are not available. A solution utilizing an additional SOGI frequency locked loop (FLL) (as a prefilter structure) to tune the APF-PLL has been proposed in [28]. However, the dynamics of FLL impacts the PLL dynamics and the paper lacks discussion on the FLL parameter design which is foremost for stable operation. Moreover, none of these techniques have examined the grid amplitude estimation procedure. Additionally, a fair comparison between frequency adaptive APF (FAAPF) and FFAPF is yet to be carried out 
to evaluate whether FFAPF offers any advantage in terms of performance.

In light of the above discussion, this paper aims to address the shortcomings in the reported FFAPF-PLL structures. Accordingly, four effective FFAPF-PLL are developed along with their small-signal model, and a comprehensive comparison among them is carried out along with the structures reported in [26]. Finally, the most efficient FFAPF version is identified, which is gauged experimentally (in different operating scenarios) with its adaptive APF (FAAPF) counterpart and two other frequency fixed PLLs using TD and SOGI as OSG units. In short, the major contributions of this paper are:

- Double harmonic oscillations present in the compensator based APF-PLL and modified transformation-based APFPLL are eliminated.

- A Notch filter-based FFAPF-PLL is proposed as an effective alternative to PR based FFAPF-PLL with improved performance.

- The detailed small-signal model (SSM), parameter tuning guidelines, and amplitude estimation approach are developed for dynamic analysis and controller synthesis.

- Comprehensive analysis of the four FFAPF PLL methods are presented by considering several performance aspects.

- Experimentally evaluating the merits/demerits of FFAPF and FAAPF.

The structure of this paper is organized as follows: The following section introduces the nonadaptive APF-PLL and derives the linearized dynamics of APF-PLL. Section III presents an in-depth analysis of four newly developed FFAPFPLL, while their comprehensive comparison is carried out in Section IV. The experimental evaluation of FFAPF, FAAPF, and two other frequency fixed PLLs from TD and SOGI families are carried out in Section V. Finally, Section VI provides concluding remarks.

\section{OVERVIEW OF NON-ADAPTIVE APF PLL}

The structure of APF-PLL is shown in Fig.1(a), where $v_{g}(t)$ is the input grid voltage showing the frequency of $\omega_{g}$. Through the APF output, a quadrature signal set $\left(v_{\alpha}, v_{\beta}\right)$ is formed, on which the Park transformation is applied using the estimated phase $\left(\theta_{e}\right)$ from the PLL itself. Proportional-integral (PI) controller is used as the loop filter while an integrator acts as the voltage-controlled oscillator. The tuning of APF can either be fixed to the nominal frequency, $\omega_{n}$ (FF), or adapted through the estimated frequency, $\omega_{e}$ (FA) from the PLL, as reflected in the figure. The transfer function of APF tuned to $\omega_{n}$ is given in (1) while its implementation structure is depicted in Fig. 1(b).

$$
F(s)=\frac{-s+\omega_{n}}{s+\omega_{n}}
$$

At the nominal frequency, the output of APF $\left(v_{\beta}\right)$ is exactly $-90^{\circ}$ the phase shifted version of $v_{\alpha}=v_{g}(t)$. However, when the grid frequency diverges from $\omega_{n}$, a phase offset error occurs resulting in non-orthogonality of the $\left(v_{\alpha}, v \beta\right)$. For a grid frequency $\omega_{g}$, the phase shift from APF-OSG becomes:

$$
\angle F\left(j \omega_{g}\right)=\tan ^{-1}\left(-\frac{2 \omega_{n} \omega_{g}}{\omega_{n}^{2}-\omega_{g}^{2}}\right)
$$

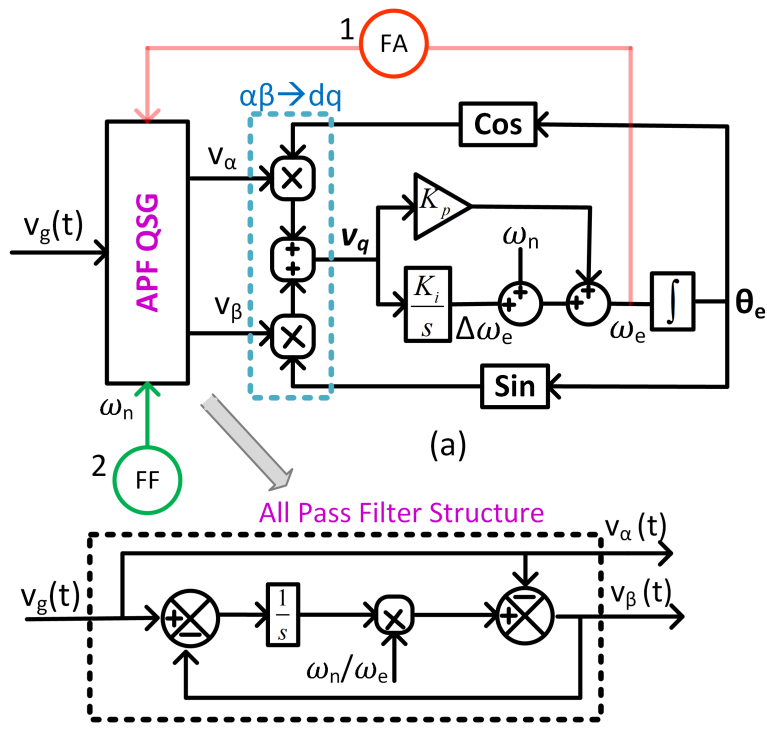

(b)

Fig. 1: Schematic of (a) APF PLL and (b) implementation of all pass filter

which can further be written, using $\tan ^{-1}\left(\frac{1}{x}\right)=-\frac{\pi}{2}-$ $\tan ^{-1}(x)$, (for $x<0$ ) as:

$$
\angle F\left(j \omega_{g}\right)=-\frac{\pi}{2}-\tan ^{-1}\left(\frac{\omega_{g}^{2}-\omega_{n}^{2}}{2 \omega_{g} \omega_{n}}\right)
$$

The grid code limits the allowable range of frequency variation (for instance, EN51600 permits $47 \mathrm{~Hz} \leq f_{\text {grid }} \leq 52 \mathrm{~Hz}$ ), and thus the phase drift of $\beta$ signal around $-\pi / 2$ due to frequency variation can be approximated as:

$$
\Delta \phi=-\tan ^{-1}\left(\frac{\omega_{g}^{2}-\omega_{n}^{2}}{2 \omega_{g} \omega_{n}}\right) \approx-\frac{\omega_{g}^{2}-\omega_{n}^{2}}{2 \omega_{g} \omega_{n}}
$$

\section{A. Linearized model for APF Dynamics}

In this section, the dynamics of the phase detector comprised of APF and $d q$ transformation is carried out. Assuming the APF-PLL is in a locked state, a sudden phase jump of $\Delta \delta$ occurs in the input signal. The generalized expression for grid voltage as a piecewise continuous signal can be written as [29]:

$v_{g}(t)=V_{m} \sin \left(\omega_{g} t\right)+u(t)\left[V_{m} \sin \left(\omega_{g} t+\Delta \delta\right)-V_{m} \sin \left(\omega_{g} t\right)\right]$

The first and second terms in the above equation are the signal just before and after the phase perturbation. For the small value of $\Delta \delta$, applying small angle sine and cosine approximation in (5) can be reduced by:

$$
v_{g}(t)=v_{\alpha}(t) \approx V_{m} \sin \left(\omega_{g} t\right)+u(t)\left[\Delta \delta V_{m} \cos \left(\omega_{g} t\right)\right]
$$

The orthogonal signal $v_{\beta}$ in s-domain (output from the APF) can be written as:

$$
V_{\beta}(s)=V_{g}(s) F(s)
$$

Taking the inverse Laplace transform, the orthogonal signal in time-domain is obtained as:

$$
v_{\beta}(t) \approx-V_{m} \cos \left(\omega_{g} t\right)+\Delta \delta V_{m}\left[\sin \left(\omega_{g} t\right)-e^{-\omega_{n} t}\right] u(t)
$$


where the first term is the steady-state output from the APF just before the occurrence of the disturbance. The second term contains the dynamics of the step phase jump. The Park $(d q)$ transformation is applied to this orthogonal component using the transformation matrix $\mathrm{T}$ given by:

$$
\left[\begin{array}{l}
v_{d} \\
v_{q}
\end{array}\right]=\underbrace{\left[\begin{array}{cc}
\sin \omega_{e} t & -\cos \omega_{e} t \\
\cos \omega_{e} t & \sin \omega_{e} t
\end{array}\right]}_{T}\left[\begin{array}{l}
v_{\alpha}(t) \\
v_{\beta}(t)
\end{array}\right]
$$

where $\omega_{e} t=\theta_{e}$ is the estimated phase of the PLL. After trigonometric simplification, the $q$ component can be derived as:

$v_{q} \approx V_{m} \sin \left(\theta_{g}-\theta_{e}\right)+\Delta \delta V_{m}\left[\cos \left(\theta_{g}-\theta_{e}\right)-\sin \left(\theta_{e}\right) e^{-\omega_{n} t}\right] u(t)$

Again, the second term in (10) shows the dynamics associated with the APF and SRF for the step phase jump after time $t=0$. Under $\theta_{e} \rightarrow \theta_{g}$ (quasi locked state), $\cos (\Delta \theta) \approx 1$ ) and assuming $\omega_{e} \approx \omega_{n}, V_{m}=1$, the Laplace transform of the second term after simplification can be expressed as:

$$
v_{q}(s) \approx \frac{\Delta \delta}{s}\left[\frac{s^{2}+\omega_{n} s+2 \omega_{n}^{2}}{s^{2}+2 \omega_{n} s+2 \omega_{n}^{2}}\right]
$$

For low frequency $\left(\omega<\omega_{n}\right)$, the above expression can further be reduced to the following first-order approximation:

$$
\frac{v_{q}(s)}{\Delta \delta(s)} \approx \frac{s+2 \omega_{n}}{2\left(s+\omega_{n}\right)}
$$

where $\Delta \delta(s)=\Delta \delta / s$ is the small-signal phase step change. The accuracy of the derived model is inspected in the next section.

\section{FREQUENCY FIXED APF-PLL}

In general, two approaches can be applied to eliminate errors originating from the FFAPF structure at offnominal frequency. First, allowing the error to propagate through the loop and compensating it either inside and/or outside of the loop. In the second method, the phase detector is modified itself for partial or complete elimination of the error component.

\section{A. Error Quantification}

This section identifies the error elements induced in the estimated $d q$ quantities because of grid frequency deviation. Consider the steady-state input signal as $v_{g}(t)=V_{m} \sin \left(\omega_{g} t\right)$, the steady state orthogonal output signals from APF tuned at $\omega_{n}$ are:

$$
\begin{aligned}
& v_{g}(t)=v_{\alpha}(t)=V_{m} \sin \left(\theta_{g}\right) \\
& v_{\beta}(t)=-V_{m} \cos \left(\theta_{g}+\Delta \phi\right)
\end{aligned}
$$

Now applying the Park transformation in (9) to the orthogonal signal set in (13):

$$
v_{q}(t)=V_{m} \sin \left(\theta_{g}\right) \cdot \cos \left(\theta_{e}\right)-V_{m} \cos \left(\theta_{g}+\Delta \phi\right) \cdot \sin \left(\theta_{e}\right)
$$

Through trigonometric simplification and applying small-angle approximation of sine and cosine for $\Delta \phi \ll 1, \sin (\Delta \phi) \approx \Delta \phi$ and $\cos (\Delta \phi) \approx 1$, the expression in (14) can be reduced to:

$$
v_{q}(t)=V_{m}\left[\sin \left(\theta_{g}-\theta_{e}\right)+\left(\frac{1-\cos \left(\theta_{g}+\theta_{e}\right)}{2}\right) \Delta \phi\right]
$$

During the quasi-locked state, the effect of this drift culminates in a phase offset error and a second harmonic oscillation in the estimated quantities.

$$
v_{q}(t) \approx V_{m}[\Delta \theta+\underbrace{\frac{\Delta \phi}{2}-\frac{\Delta \phi}{2} \cos \left(2 \theta_{e}+\Delta \theta\right)}_{\text {error }}]
$$

The error term in (16) depends on the grid voltage amplitude. To discard this dependency, the $v_{q}$ signal can be normalized with the estimated magnitude from $v_{d}$. Using the orthogonal signal set $\left(v_{\alpha}, v_{\beta}\right)$ and transformation matrix in (9), the $d$ component is calculated as:

$$
v_{d}=V_{m}\left[\sin \left(\theta_{e}\right) \cdot \sin \left(\theta_{g}\right)+\cos \left(\theta_{g}+\Delta \phi\right) \cdot \cos \left(\theta_{e}\right)\right]
$$

Through trigonometric simplification and utilizing the smallangle approximation, $v_{d}$ can be expressed as:

$$
v_{d}(t) \approx V_{m}\left[1-\frac{\Delta \phi}{2} \sin 2 \theta_{e}\right]
$$

Consequently, the estimated amplitude also includes an oscillatory error of twice the fundamental frequency when the grid frequency diverges

\section{B. Compensator Based FFAPF}

If $v_{q}$ can be normalized with the magnitude $V_{m}$, and phase lock condition arises $\left(\theta_{e}\right.$ close to $\left.\theta_{g}\right)$ both errors can be eliminated with additional structure. The estimated $d$ axis component $\left(v_{d}\right)$ is first passed through two repeated delayed signal cancellation (DSC) of delay length $\left(N_{d}=\left(T_{n} / 4\right) / T_{s}\right)$ for attenuating $2 \omega_{e}$ component with wide stretch around this frequency to obtain $v_{d f} . T_{n}$ and $T_{s}$ are the nominal grid period and sampling frequency, respectively.

The phase offset error can be negated at the PLL output (phase), by adding the estimated error in the opposite sign as below:

$$
\theta_{\text {corr }} \approx \theta_{e}-\frac{\Delta \phi_{e}}{2}
$$

$\Delta \phi_{e}$ can be estimated using the expression in (4). The second harmonic component is approximated (using the estimated phase $\theta_{e}$ ) and then injected (with opposite sign) into calculated $v_{q}$ component before PI input:

$$
v_{q c o r r}(t) \approx v_{q}(t)+\frac{\Delta \phi_{e}}{2} \cos \left(2 \theta_{e}\right)
$$

The detailed execution of this approach is shown in Fig. 2, where ANM is the amplitude normalization module responsible for implementing $\left(v_{q} / v_{d f}\right)$. This PLL is hereafter referred to as compensator and cancellation-based APF (CCAPF) PLL.

APF and PLL control loops are decoupled and hence APF dynamics remains outside of the control loop. To account the effect of phase error compensator, consider a small frequency drift of $\Delta \omega_{g}=\omega_{g}-\omega_{n}$ and $\omega_{g}$ close to $\omega_{n}$, then $\Delta \phi$ in (4) can be approximated as:

$$
\Delta \phi=\left(\frac{-\Delta \omega_{g}\left(\omega_{g}+\omega_{n}\right)}{2 \omega_{g} \omega_{n}}\right) \approx-\left(\frac{\Delta \omega_{g}}{\omega_{n}}\right)
$$




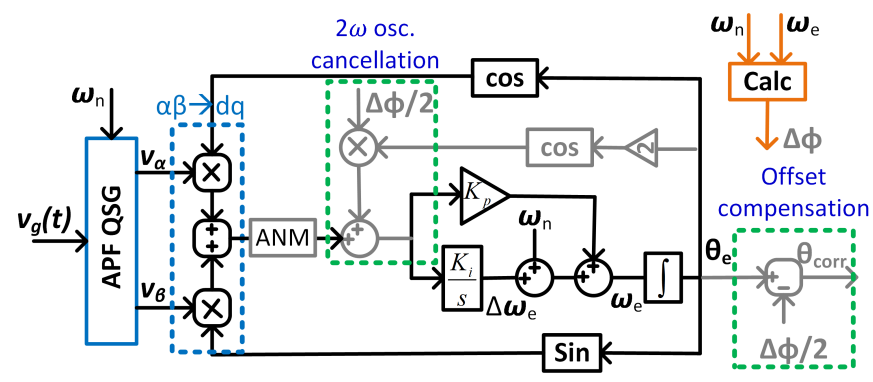

Fig. 2: Compensator and cancellation network based frequency fixed APF-PLL

Hence, the phase shift $(\Delta \phi)$ arising because of variation of $\omega_{g}$, is linearly related to $\Delta \omega_{g}$, which can be obtained from output of the integrator. The SSM for the phase jump of $\Delta \theta_{g}$ on grid phase $\theta_{g}$ can then be derived as shown in Fig.3(a).

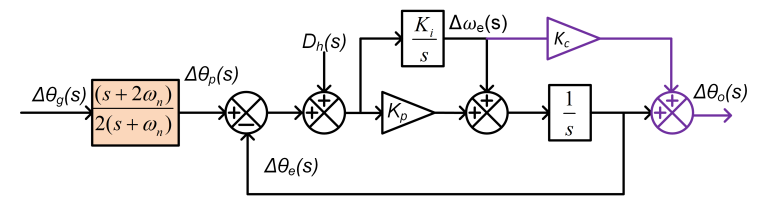

(a)
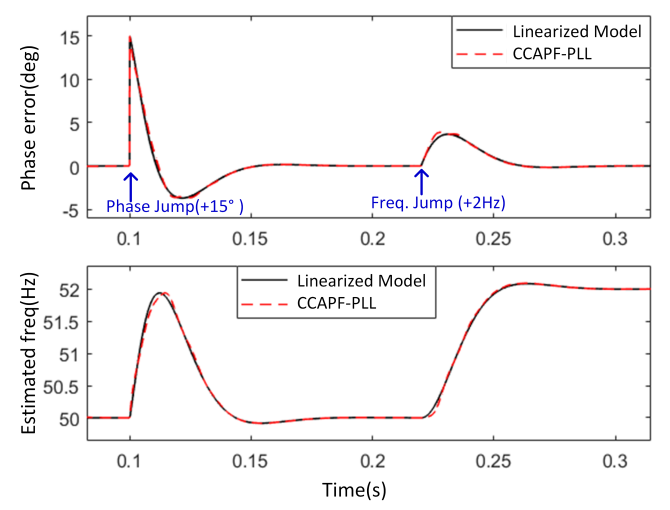

(b)

Fig. 3: (a)The SSM control block diagram of CCAPF-PLL (b) Simulation results for verifying accuracy of derived SSM

The assessment of the derived small-signal model is presented in Fig. 3(b). A phase jump of $+15^{\circ}$ (at $0.1 \mathrm{~s}$ ) and a frequency jump of $+2 \mathrm{~Hz}$ (at $t=0.22 \mathrm{~s}$ ) is applied to both models (CCAPF-PLL and small) and with same control parameters $\left(K_{p}=151, K_{i}=11409, K_{c}=1 / 2 \omega_{n}\right)$. It is visible that the linearized model predicts the estimation behaviour of both phase and frequency with high precision.

From the linearized model, it is clear that APF and SRFPLL are isolated. The open-loop transfer function, while neglecting the feedforward path, can be written as:

$$
\frac{\Delta \theta_{e}(s)}{\Delta \theta_{p}(s)-\Delta \theta_{e}(s)}=\frac{1}{s} \cdot \frac{K_{p} s+K_{i}}{s}
$$

The final closed-loop transfer function can thus be expressed as:

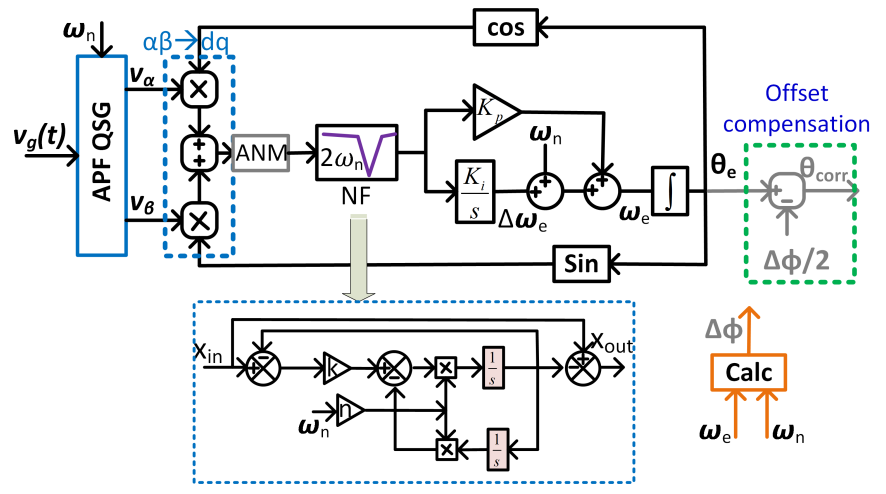

Fig. 4: NF and compensator based fixed frequency APF-PLL

$$
\frac{\Delta \theta_{o}(s)}{\Delta \theta_{g}(s)}=\frac{0.5\left(s+2 \omega_{n}\right)}{s+\omega_{n}} \frac{\left(K_{p}+K_{c} K_{i}\right) s+K_{i}}{s^{2}+K_{p} s+K_{i}}
$$

Comparing the denominator of the second multiplicative term in (23) with the standard $2^{\text {nd }}$ order characteristic equation $\left(s^{2}+2 \zeta \omega_{n f}+\omega_{n f}^{2}\right)$, we can obtain $\left(K_{p}=2 \zeta \omega_{n f}\right)$ and $\left(K_{i}=\omega_{n f}^{2}\right)$, where $\zeta$ (damping factor) and $\omega_{n f}$ (natural frequency) determine the performance of the closed-loop system. $\zeta$ is usually taken as $1 / \sqrt{2}$ for optimum damping performance, while $\omega_{n f}$ is another design parameter influencing crossover frequency (settling time) and filtering capability. Ensuing the tradeoff between bandwidth and filtering, a choice of $\omega_{n} f=2 \pi 20 \mathrm{rad} / \mathrm{s}$ returns the controller parameters at $K_{p}=178$ and $K_{i}=15791$.

\section{Notch Filter based FFAPF}

The inclusion of a notch filter (NF) tuned to $2 \omega_{n}$ is a straightforward approach to deal with the oscillatory error inside PLL control loop, as illustrated in Fig. 4. Meanwhile, the dc phase error component is removed via the compensator network exactly as the solution for CCAPF discussed in section III-B. Thus, the resultant PLL is named as the notch and compensator FFAPF (NCAPF)-PLL. The transfer function of the nonadaptive NF is given as (24):

$$
G_{n f}(s)=\frac{s^{2}+\left(2 \omega_{n}\right)^{2}}{s^{2}+k_{n}\left(2 \omega_{n}\right) s+\left(2 \omega_{n}\right)^{2}}
$$

where, $k_{n}$ is the inverse of NF quality factor, which determines the notch width as well as the phase lag it confers at low frequency. It is chosen as $\sqrt{2}$ for optimum performance. This selection also provides acceptable blockage of the double frequency component even in grid frequency variation. However, if higher accuracy is desired, an adaptive NF (fed by the low pass filtered estimated frequency) maybe used to tune the resonant frequency to the estimated frequency. Such an additional feedback results in dual-loop systems with the frequency loop residing internal to the phase feedback loop. ANM can be integrated inside the control loop similarly as in the CCAPF. An additional NF $\left(2 \omega_{n}\right)$ can be placed in amplitude $\left(v_{d}\right)$ estimation path. 
The controller parameters are affected by the NF which resides inside the loop. To simplify the controller design, a first-order approximation of NF as given in (25) is used [30]:

$$
G_{n f}(s) \approx \frac{1}{\left(k_{n} / 2 \omega_{n}\right) s+1}
$$

The small signal model for NCAPF can be obtained as

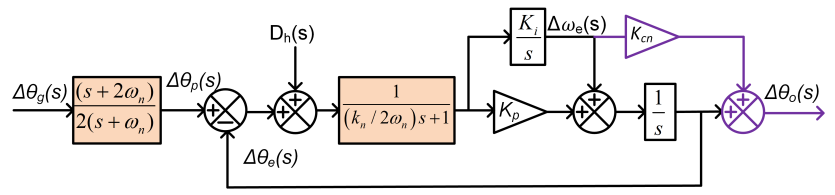

(a)

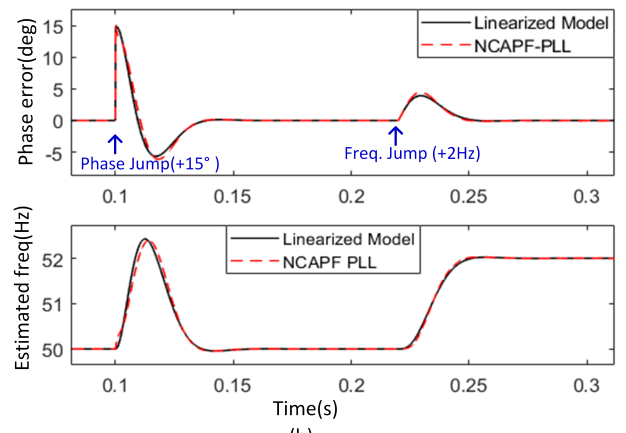

(b)

Fig. 5: (a)Small-signal model of FFAPF-PLL using NF (b) accuracy assessment of the derived SSM

depicted in Fig. 5. The feedforward network is again identical to that of CCAPF. The accuracy of the derived model is verified by performance comparison with NCAPF through simulation results. The same control parameters are used for both, and the results (phase error and frequency estimation) in Fig. 5(b) clearly indicate that the model provides an accurate prediction of NCAPF-PLL behaviour. The open-loop transfer function, $\theta_{e}(s) /\left(\theta_{e}(s)-\theta_{p}(s)\right)$ (ignoring the feedforward correction) can be written as:

$$
G_{o \ln }(s) \approx \underbrace{\frac{1}{\left(k_{n} / 2 \omega_{n}\right)} s+1}_{T_{d}} \cdot\left(\frac{K_{p} s+K_{i}}{s}\right) \cdot \frac{1}{s}
$$

For type II open loop transfer function as in (26), the symmetric optimum method has been applied to design the controller parameters [23], [31]. The proportional and integral gains are a function of a design constant $\mu$ and $T_{d}$. The value of $\mu$ is related to the phase margin $\left(\phi_{p m}\right)$ and given as:

$$
\mu=\frac{1+\sin \left(\phi_{p m}\right)}{\cos \left(\phi_{p m}\right)} \&\left\{\begin{array}{l}
K_{p}=\frac{1}{\mu T_{d}} \\
K_{i}=\frac{1}{\mu^{3} T_{d}{ }^{2}}
\end{array}\right.
$$

Usually PM in the range of $30^{\circ}<\phi_{p m}<60^{\circ}$ is desirable and for a choice of $45^{\circ}$, it yields $K_{p}=184$ and $K_{i}=14111$, while, $k_{c n}$ is still $1 / 2 \omega_{n}$. Based on the analysis from the previous section, the final closed-loop transfer function can be derived as:

$\frac{\Delta \theta_{o}(s)}{\Delta \theta_{g}(s)}=\frac{0.5\left(s+2 \omega_{n}\right)}{s+\omega_{n}} \cdot \frac{G_{o \ln }(s)}{1+G_{o \ln }(s)} \cdot \frac{\left(K_{p}+K_{c n} K_{i}\right) s+K_{i}}{\left(K_{p} s+K i\right)}$

\section{Two Stage APF}

Inspired from [32], the input signal is passed successively through consecutive stages of APF to obtain $v_{\alpha}$ signal as shown in Fig. 6. The operation of this mechanism, two-stage APF (TSAPF) is explained from a frequency domain point of view in [26], while here the functioning is presented from time domain perspective. Consider the input voltage signal $v_{g}(t)=V_{m} \sin \left(\omega_{g} t\right)$ ), while its steady state $v_{\beta}(t)$ signal is given in (13). When $\left(v_{g}(t)\right)$ is again passed through another APF, the resultant signal is obtained as:

$$
v_{g}^{\prime}(t)=-V_{m} \sin \left(\omega_{g} t+2 \Delta \phi\right)
$$

Through small-angle approximation and simplification, the new $v_{\alpha}$ component can be approximated as:

$$
v_{\alpha}=\left(v_{g}(t)-v_{g}{ }^{\prime}(t)\right) / 2 \approx V_{m} \sin \left(\theta_{g}\right)+\Delta \phi \cos \left(\theta_{g}\right)
$$

Then, $v_{q}$ component is calculated by substituting orthogonal signals $\left(v_{\alpha}\right.$ in (30), $v_{\beta}$ in (13)) for the transformation in (9). After some trigonometric simplification and small-angle approximation, the final expression for $v_{q}$ becomes:

$$
v_{q} \approx V_{m} \sin \left(\theta_{g}-\theta_{e}\right)+\Delta \phi \cos \left(\theta_{g}-\theta_{e}\right)
$$

When the estimated phase $\left(\theta_{e}\right)$ is closer to the grid phase $\left(\theta_{g}\right)$, the $v_{q}$ signal contains additional phase offset error of $\Delta \phi$. Compared to single-stage FFAPF, the double frequency component is absent, hence only the phase error correction is sufficient as reflected in Fig. 6. Here, the gain of the phase error compensator is twice of the gain in CCAPF-PLL. It should be pointed out that $\Delta \phi$ is calculated using expression (4), unlike in [26] where the small signal value (21) was used in the actual PLL. This improves the reduction of phase offset error in large frequency drifts.

For the amplitude estimation, consider the same orthogonal signals and the transformation matrix (9). Substituting the $\alpha, \beta$ signals, after some trigonometric simplification and applying small-angle approximation, the following expression can be obtained:

$$
v_{d} \approx V_{m}\left(\cos \left(\theta_{g}-\theta_{e}\right)\right)-\Delta \phi\left(\sin \left(\theta_{g}-\theta_{e}\right)\right)
$$

When $\theta_{e} \rightarrow \theta_{g}$, the signal $v_{d}$ converges to grid amplitude $V_{m}$. Thus, no separate structure is required for correcting the amplitude estimation.

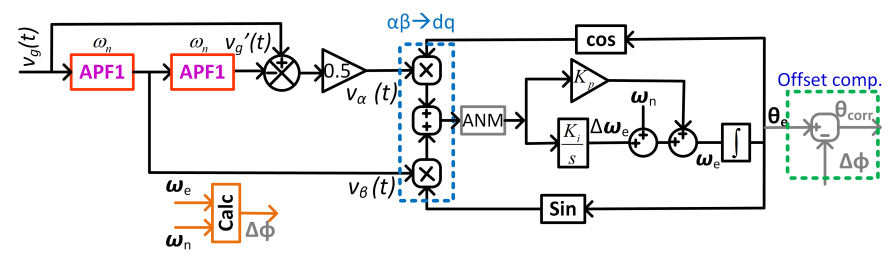

Fig. 6: Two stage fixed frequency APF-PLL structure with phase compensator

The small signal model of TSAPF can be obtained, as shown in Fig. 7(a). It is similar to that of the single stage except the inclusion of another APF stage before the PLL control 
loop and the gain of the phase compensator. The open-loop transfer function is the same as for single-stage FFAPF when the feedforward controller is neglected. The final closed-loop transfer function (output phase to input phase) again can be derived following the same procedure as for CCAPF-PLL as below.

$$
\frac{\Delta \theta_{o}(s)}{\Delta \theta_{g}(s)}=\left(\frac{0.5\left(s+2 \omega_{n}\right)}{s+\omega_{n}}\right)^{2} \frac{\left(K_{p}+K_{r} K_{i}\right) s+K_{i}}{s^{2}+K_{p} s+K_{i}}
$$

The verification of the derived model is carried out by comparing the simulation results of TSAPF-PLL and its SSM using the same control parameters. The results (estimated phase and frequency) in Fig. 7(b), clearly exhibit that the model accurately mimics the dynamic behaviour of TSAPFPLL.
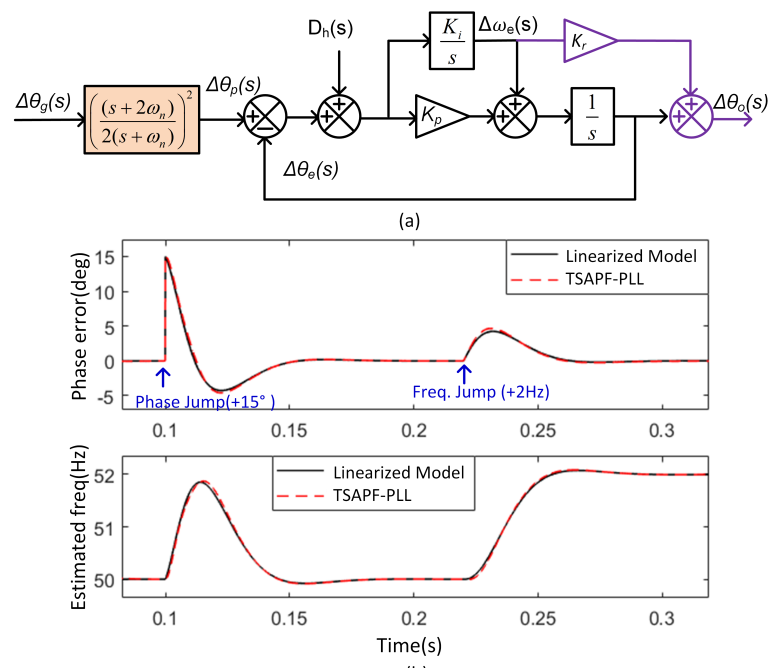

(b)

Fig. 7: (a)SSM of TSAPF-PLL (b) Accuracy assessment of SSM model

The control parameter design follows the same procedure as that for CCAPF and for the sake of brevity, it is not repeated here. For fair comparison, the same design criteria are used giving the same control parameters $\left(K_{p}=178\right.$ and $\left.K_{i}=15791\right)$.

\section{E. Modified Transformation FFAPF}

Consider again the $v_{q}$ calculation using fixed frequency APF as below:

$$
v_{q}(t)=v_{\alpha} \cdot \cos \left(\theta_{e}\right)+v_{\alpha}\left(\theta_{g}-\phi_{\omega_{n}}\right) \cdot \sin \left(\theta_{e}\right)
$$

where, $\phi_{\omega_{n}}$ is phase shift from APF tuned to $\omega_{n}$ for $\beta$ component. At $\omega_{g} \neq \omega_{n}$, an error component occurs in the second term of the above expression. The idea is to generate an equal and opposite error in the first term by placing another nonadaptive APF block for indirect generation of the cosine of the estimated angle. This method is inspired from the nonfrequency dependent time delay PLL (NTD-PLL) [33] and here labelled as modified transformation APF (MTAPF). Both the APFs are tuned to a nominal frequency of $\omega_{n}$ such that they provide same phase shift during quasi locked state when $\omega_{e}$ approaches $\omega_{g}$. The mathematical expression for such realization is given below while the structural modification is depicted in Fig. 8.

$v_{q}(t)=-v_{\alpha}\left(\omega_{g} t\right) \cdot \underbrace{\sin \left(\omega_{e} t-\phi_{\omega n 2}\right)}_{A P F 2}+\sin \left(\omega_{e} t\right) \cdot \underbrace{v_{\alpha}\left(\omega_{g} t-\phi_{\omega n 1}\right)}_{A P F 1}$

where, $\phi_{\omega_{n x}}$ is the phase shift obtained from $A P F_{x}$ tuned at $\omega_{n}(50 \mathrm{~Hz})$ and $\omega_{e}$ is the estimated frequency from PLL. Let $\omega_{g} \neq \omega_{n}$ and $\Delta \phi_{x}$ be the phase displacement because of grid frequency drift. Then, the above equation can be rewritten as:

$=\underbrace{-\overbrace{V_{g} \sin \left(\theta_{g}\right)}^{v_{\alpha}} \cdot \overbrace{\cos \left(\theta_{e}+\Delta \phi_{2}\right)}^{A P F 2}}_{\text {Term }-1}+\underbrace{\sin \left(\theta_{e}\right) \cdot \overbrace{V_{g} \cos \left(\theta_{g} t+\Delta \phi_{1}\right)}^{\left(v_{\beta}\right) A P F 1}}_{\text {Term }-2}$

Equation (36) reveals irrespective of $\omega_{n}, \omega_{e}=\omega_{g}$ is sufficient for impelling $\Delta \phi_{1}=\Delta \phi_{2}$ which results in the cancellation of error components generated from each term. Thus, $v_{q}$ can be regulated to zero. This is contrary to the reported work in [26], where APF was employed in the second term of expression in the right hand side to indirectly generate $\sin \left(\omega_{e} t\right)$ and hence could only eliminate the offset error while the second harmonic oscillations still remained .

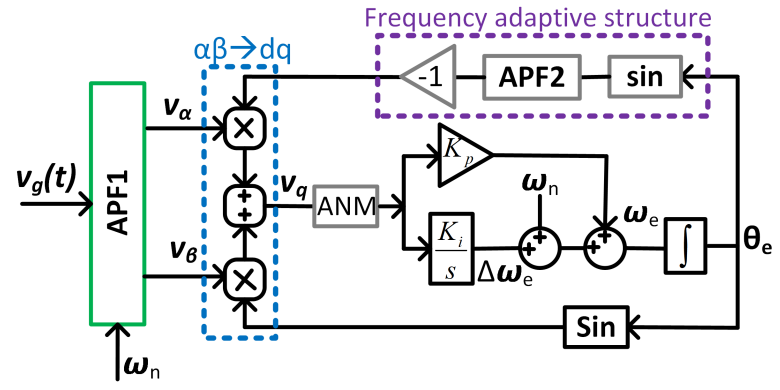

Fig. 8: Modified transformation APF-PLL to incorporate frequency adaptive feature

Similarly, the ANM based on the modified DQ transformation can be integrated to avoid the dependence of the open loop gain on the grid voltage amplitude. Modified DQ transformation can also be applied for the estimation of grid voltage magnitude. Applying a similar concept, the error present in the second term of the expression (17) is reintroduced in its first term. This is achieved by replacing $\sin \left(\theta_{e}\right)$ with $\cos \left(\theta_{e}\right)$ in (17) which passes through another fixed frequency APF block tuned at $\omega_{n}$.

$$
v_{d}=V_{m}[\underbrace{\sin \left(\theta_{e}+\Delta \phi\right)}_{\text {APFout }} \cdot \underbrace{\sin \left(\theta_{g}\right)}_{v_{\alpha}}+\underbrace{\cos \left(\theta_{g}+\Delta \phi\right)}_{v_{\beta}} \cdot \cos \left(\theta_{e}\right)]
$$

Expanding the above expression and through some assumptions $\left(\theta_{e}\right.$ closer to $\left.\theta_{g}\right)$ and trigonometric processing, the magnitude can be estimated as:

$$
V_{m} \approx \frac{v_{d}}{\cos (\Delta \phi)}
$$

For small grid frequency variation, $\cos (\Delta \phi) \rightarrow 1$, and the effect of division term is tiny in the estimated quantities and 
can be neglected. Alternately, the Taylor series approximation of the cosine up to two terms can be used to retain high accuracy without inflating implementation.:

$$
\cos (\Delta \phi) \approx 1-\left(\left(\Delta \phi^{2}\right) / 2\right)
$$

In MTAPF-PLL, although there is an absence of frequency feedback, an additional APF lies in the phase feedback path instead. This consequently introduces the APF delay effect in the PLL control loop. Hence, the small signal model of the modified transformation-based APF-PLL is same as that of the frequency adaptive APF (FAAPF) PLL and is shown in Fig. 9 (a). This means, despite having a structurally different implementation, from the small signal perspective, they are equivalent.

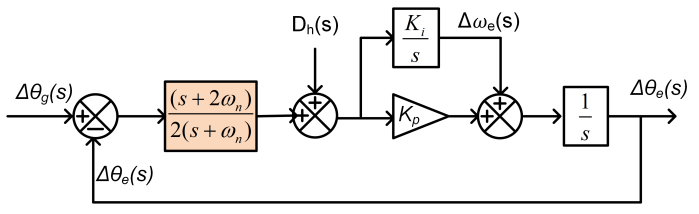

(a)
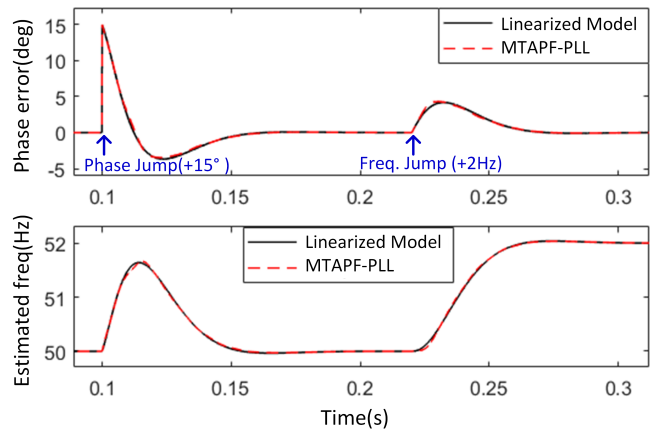

(b)

Fig. 9: (b) Linearized model of modified dq transformation based APF-PLL and (b) Accuracy verification of derived model in phase and frequency jump

The correctness of the linearized model (MTAPF-PLL) presented above is justified via simulation test. A phase jump of $+15^{\circ}$ and a frequency jump of $+2 \mathrm{~Hz}$ are applied, and the corresponding phase error and estimated frequency are depicted in Fig. 9(b). The results clearly show that the derived model provides an accurate approximation of the actual MTAPF-PLL.

To eliminate the effect of grid voltage amplitude on the controller parameter design, the $v_{q}$ can be normalized with $v_{d}$ as discussed beforehand. If the proportional and integral gains are $K_{p}$ and $K_{i}$, the open-loop transfer function of MTAPFPLL with PI controller is given as:

$$
G_{o l}(s)=\frac{\Delta \theta_{e}(s)}{\Delta \theta_{g}(s)-\Delta \theta_{e}(s)}=\underbrace{\frac{\left(s+2 \omega_{n}\right)}{2\left(s+\omega_{n}\right)}}_{v_{q}(s)} \underbrace{\left(\frac{K_{p} s+K_{i}}{s}\right)}_{G_{p i}(s)} \cdot \frac{1}{s}
$$

where $v_{q}(s)$ is transfer function representing MTAPF-PD dynamics and $G_{p i}(s)$ is PI controller transfer function. The transfer function of $v_{q}(s)$ is a lag filter filter and in low frequency range, the effect of this filter can be overlooked while maintaining reasonable accuracy [10]. This simplifies the controller design procedure as the closed-loop transfer function can be expressed in standard form below:

$$
G_{c l}(s)=\frac{\Delta \theta_{e}(s)}{\Delta \theta_{g}(s)}=\frac{K_{p} s+K_{i}}{s^{2}+K_{p} s+K_{i}}
$$

Comparing the above closed-loop transfer function with the standard second-order transfer function with zero, the controller gains can be equated as $K_{p}=2 \zeta \omega_{n}^{\prime}$ and $K_{i}=\omega_{n}^{\prime 2}$ respectively. The controller design follows the same approach as discussed for CCAPF-PLL. For an impartial comparison with other FFPLLs $\left(\zeta=0.707, \omega_{n}^{\prime}=20 \pi\right)$, the choice of controller parameter must be consistent as $K_{p}=178$ and $K_{i}=15791$.

\section{Comparative Analysis of FFAPF-PLL}

In this section, the performances of the four FFAPF-PLL are evaluated in four facets, namely, dynamics, accuracy, implementation, and disturbance rejection. The controller parameters used are as designed in the aforementioned section using the standard stability criterion for a fair comparison. The bode plot of the compensated loop gain for the four approaches along with their phase margin (PM) and crossover frequency is illustrated in Fig. 10. For MTAPF, the obtained PM is lower as the phase contribution from APF was omitted during the controller design. Although small, in reality, it does present some amount of phase lag at around the frequency of concern $\left(\omega_{c}\right)$.

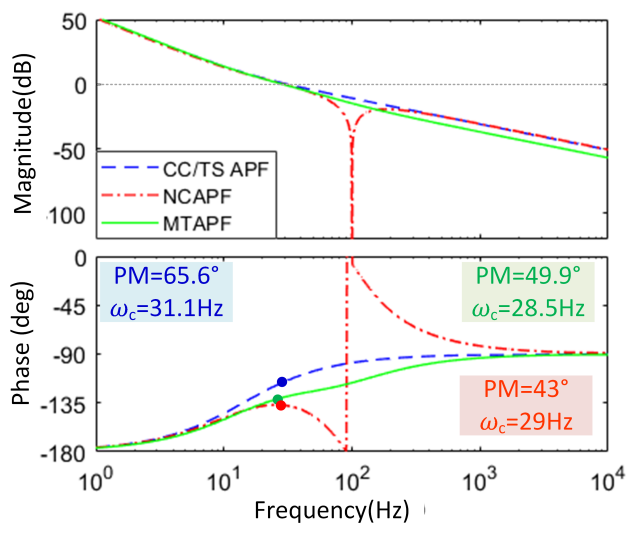

Fig. 10: Bode plot of compensated loop gain of four FFAPFPLL

\section{A. Dynamic Performance}

The dynamic responses of four FFAPF-PLLs are evaluated under a phase jump of $\left(+30^{\circ}\right)$ and a frequency step change of $(+2 \mathrm{~Hz})$. The $2 \%$ settling time, percentage overshoot, and peak phase/frequency error are appraised as performance metrics. Moreover, the maximum amplitude variation without saturation unit is also noted. The summary of the measurement is presented in Table I while simulation figures are omitted to save space. The results reflect the fast dynamic performance 
TABLE I: Dynamic performance assessment of four FFAPFPLLs

\begin{tabular}{lllll}
\hline Disturbance & CCAPF & NCAPF & TSAPF & MTAPF \\
\hline Phase jump $\left(+30^{\circ}\right)$ & & & & \\
2\% settling time $(\mathrm{ms})$ & 41.34 & 35.146 & 42.47 & 34.378 \\
Phase overshoot $(\%)$ & 26.08 & 42.03 & 32.53 & 32.13 \\
Peak Freq. error (Hz) & 4.48 & 4.832 & 4.28 & 4.93 \\
Peak Amp. error(p.u) & 0.22 & 0.464 & 0.4509 & 0.4845 \\
\hline Freq. step (+2Hz) & & & & \\
2\% settling time (ms) & 48.52 & 27.228 & 50.48 & 40.965 \\
Freq. overshoot (\%) & 4.25 & 1.316 & 4.35 & 3.95 \\
Peak phase error (deg) & 3.41 & 4.327 & 4.10 & 3.51 \\
Peak Amp. error(p.u) & 0.004 & 0.006 & 0.006 & 0.013 \\
\hline $\mathbf{- 3 d B}, \theta_{o}(s) / \theta_{g}(s)(\mathrm{Hz})$ & 39.1 & 45.8 & 37.4 & 42.8 \\
\hline
\end{tabular}
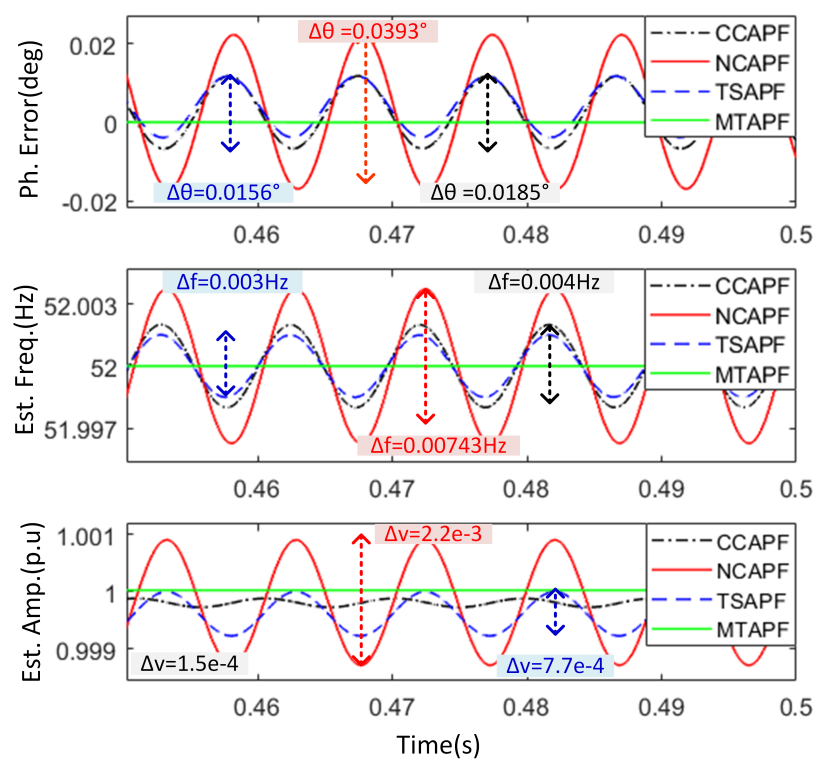

Fig. 11: Variation of the estimated quantities around the final steady state value

of NCAPF and MTAPF. While, CCAPF and TSAPF both have comparable dynamics, with CCAPF having slightly better transient speed and lower overshoot. The measured bandwidth of closed-loop transfer function $\left(\theta_{o}(s) / \theta_{g}(s)\right)$ are also reported in table I. CCAPF meanwhile, shows the least variation of amplitude during phase/frequency disturbance.

\section{B. Steady State Performance}

The steady-state accuracies of the four FFAPF methods are analysed by measuring the steady state deviation of the estimated quantities at off-nominal frequency $(52 \mathrm{~Hz})$. The measurements of peak-to-peak oscillations are shown in Fig 11. From the results, it can be concluded that MTAPF provides the best steady state performance, free of oscillation. Meanwhile, CCAPF and TSAPF provide a similar level of precision. The NCAPF has the largest oscillation because of its weakened rejection capacity at off-nominal frequency. As discussed, if desired, this can be sorted out by adapting the notch frequency to the estimated frequency.

The improvement in SSA at off-nominal frequency brought out by the proposed MTAPF, TSAPF, and CCAPF from its
TABLE II: Number of additional operations required for implementation of each FFAPF-PLL

\begin{tabular}{lllll}
\hline Method & $\begin{array}{l}\text { Additional } \\
\text { structure }\end{array}$ & $\begin{array}{l}\text { Trig. } \\
\text { functions }\end{array}$ & $* / *$ & $+/-$ \\
\hline CCAPF & DSC(2) & 1 & 3 & 2 \\
\hline TSAPF & APF (1) & 0 & 2 & 2 \\
\hline NCAPF & NF (2) & 0 & 2 & 2 \\
\hline MTAPF & APF(2) & 0 & 4 & 2 \\
\hline
\end{tabular}

conventional version can be better visualized in the comparative simulation result depicted in Fig.12. As noted, even at $\omega_{g}=52 \mathrm{~Hz}$, the proposed MTAPF and CCAPF considerably lessen the oscillatory error in estimated phase contrary to previously recommended methods in [26]. This improvement is achieved without compromise to the dynamic performance which remains the same. Meanwhile, the proposed TSAPF gets rid of the small phase offset error with respect to the previous version in [26]. It should be pointed out that the previous studies also did not delve into measuring the amplitude estimation accuracy.

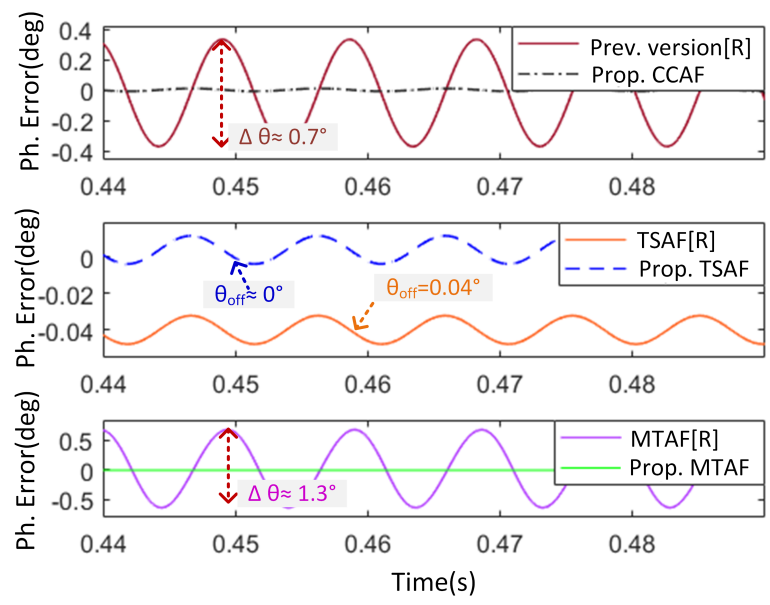

Fig. 12: Simulation results(phase error) for conventional and proposed version of FFAPF at the off-nominal frequency (52 $\mathrm{Hz}) ;([\mathrm{R}]=[26])$

\section{Computational Requirements}

The additional mathematical calculations involved in implementing the four FFAPF-PLLs are presented in Table II. The common mathematical operations such as controller, OSG and transformation, and amplitude normalization are excluded in the table. However, the supplementary structures involved in the magnitude estimation process are taken into account. The constants are lumped on one side and for MTAPF, the cosine term in (39) is replaced with its low cost implementation. From the implementation point of view, the TSAPF attains FF operation with the least additional computations

\section{Disturbance Rejection Feature}

The DC offset and harmonics appear as disturbance $\left(d_{h}(s)\right)$ input to LF after the phase detector section. DC offset in PLL input signal converts to the fundamental frequency component 


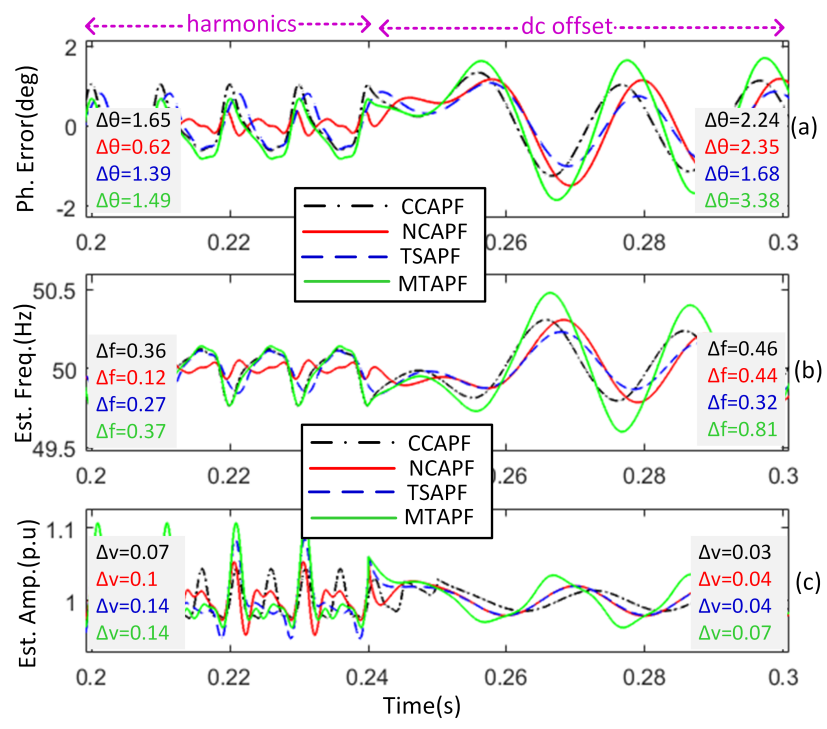

Fig. 13: The disturbance rejection capability of four FFAPF for input signal containing dc offset and low order harmonics

while the lowest harmonic, i.e, $3^{\text {rd }}$ order transforms into $2^{\text {nd }}$ and $4^{\text {th }}$ harmonic component in $d q$ frame. All PLLs are affected by these disturbances, albeit differently because of their different attenuation strengths at these frequencies. These are clarified in Fig.13 by observing the oscillatory error in the estimated quantities in the presence of dc offset $(0.02 p . u$, for $t>0.24 s)$ and harmonics $\left(3^{\text {rd }}\right.$ of $0.04 p . u$, $5^{\text {th }}$ of $0.037^{\text {th }}$ of 0.02 p.u for $t<0.24 s$ ) respectively. As the phase and frequency are estimated from the same control loop, they show similar estimation behaviour. To be precise, NCAPF as anticipated provides the best harmonic rejection and TSAPF better rejects the dc offset than rest of the PLLs as evidenced by lower peak-to-peak oscillatory errors. Meanwhile, the amplitude which is estimated separately $\left(v_{d}\right.$ signal) is also affected because of distortion and dc offset in grid voltage. Among these PLLs, CCAPF has the smallest peak-to-peak amplitude errors in both types (dc offset and harmonics) of disturbances as shown in Fig.13 (c). This is because it has a pair of DSCs tuned to $100 \mathrm{~Hz}$ placed in $d$ rotating frame.

It is noted that, in both cases (dc offset and distortion) TSAPF offers better rejection performance than CCAPF. This is because these disturbance components, when converted to a rotating frame, are reduced in magnitude in TSAPF because of the employed structure.

\section{EXPERIMENTAL ANALYSIS}

The dynamic analysis showed that among FFAPF, the MTAPF fares overall better in dynamic response for the phase and frequency estimation. It possesses the best SSA, while not being too computational intensive. The performance of MTAPF-PLL is appraised experimentally in this section using the dSPACE (DS1104) platform with frequency adaptive APFPLL (FAAPF) presented in [10]. As MTAPF and FAAPF have the same SSM, it is useful to observe the difference in their
TABLE III: Control Parameters used for experimental study

\begin{tabular}{lllll} 
Method & $\mathbf{K p}$ & $\mathbf{K i}$ & $\mathbf{k}$ & Td \\
\hline FAAPF & 178 & 15791 & - & - \\
\hline FFSOGI & 178 & 15791 & 1.41 & - \\
\hline NTD & 165 & 11431 & - & $T_{n} / 4$ \\
\hline MTAPF & 178 & 15791 & - & -
\end{tabular}

performance. Two other FF-PLLs from different OSG (TD SOGI) classes are also selected to study the competitiveness of the MTAPF with other PLL types. Among the reported FFPLLs for TD and SOGI, nonfrequency dependent TDPLL (NTD-PLL) [33] and frequency fixed SOGI (FFSOGI) in [20] are the best performing ones. The relevant control parameters for these four PLL types maintaining the standard stability criterion are listed in Table III. Three of them have the same control parameters as they have identical characteristics equations of closed-loop transfer function. While, for NTDPLL, which has different transfer functions, the suggested control parameters in the paper provide the best response. The grid voltage signals are programmed internally and generated using dSPACE itself. The sampling frequency is chosen as $10 \mathrm{KHz}$ throughout. The estimated frequency is fed back in FFSOGI to adjust the beta signal gain, while in FAAPF, for adapting the APF block. To avoid the algebraic loop, the frequency is first passed through LPF or a unit sample delay. Six test cases are conducted with different disturbances of the input voltage signal as listed below:

- Case I: Phase jump of $+30^{\circ}$

- Case II: Frequency step of $+2 \mathrm{~Hz}$

- Case III: Input voltage sag of 0.1p.u

- Case IV: Presence of dc offset (0.02p.u)

- Case V: Distorted with odd order harmonics.

- Case VI: Frequency variation with distorted grid voltage.

Fig. 14 illustrates the dynamic response of four PLLs for case I, when the input grid voltage has a sudden phase jump of $+30^{\circ}$. The tracking of the grid phase by MTAPF-PLL is the fastest, followed by NTD-PLL. The dynamics of frequency and amplitude estimation for MTAPF and FAAPF are similar with both exhibiting high overshoot, while FFSOGI has the lowest variation (peak error). Similarly, the dynamics and steady state performance for case II during grid frequency variation is depicted in Fig. 15. The NTD followed by MTAPF takes the minimum time to settle to the target frequency of $52 \mathrm{~Hz}$. MTAPF and FAAPF both demonstrate similar amounts of peak phase and magnitude estimation error during transients and lower than FFSOGI and NTD. The zoomed in version of the steady state value at off-nominal frequency $(52 \mathrm{~Hz})$ clearly reveals the superior accuracy obtained from MTAPF and NTD-PLL. FFSOGI has the largest phase and amplitude tracking error. Although tiny, FAAPF also possess fundamental frequency oscillatory components in the estimated quantities. In case III, a voltage sag of $-0.1 p . u$ is considered and the corresponding response is shown in Fig. 16. FAAPF tracks the new amplitude rapidly, followed by MTAPF. However, MTAPF settles out the transient phase/frequency error the fastest. NTD-PLL presents an identical response as that of MTAPF, and FFSOGI exhibits the slowest dynamic speed and 
higher peak errors in phase and frequency.

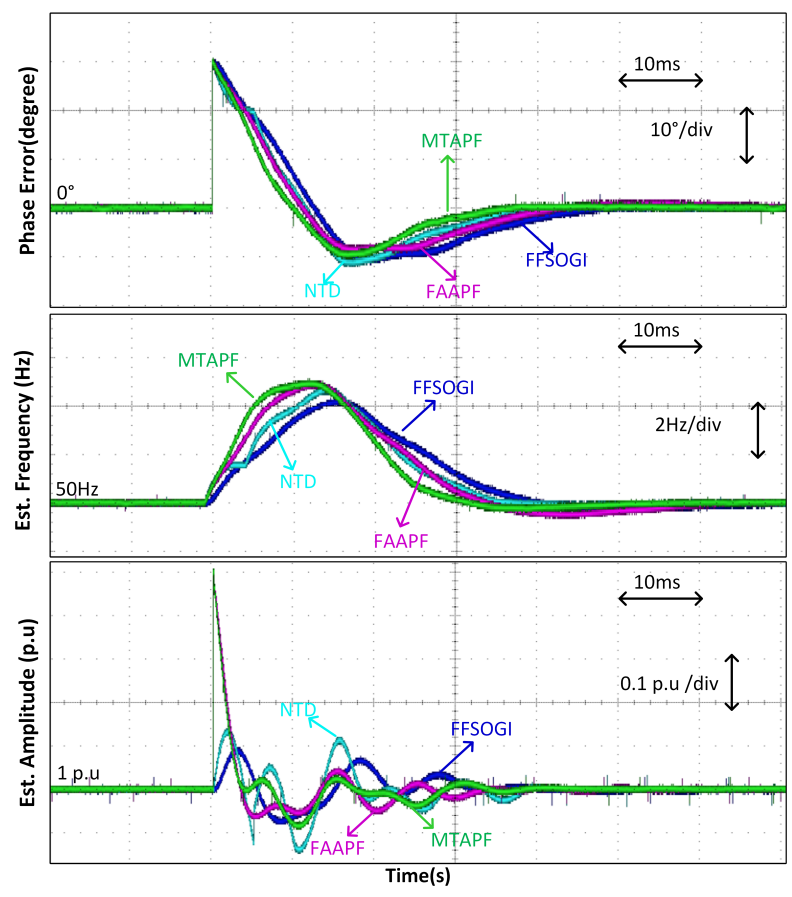

Fig. 14: Dynamic performance in event of phase jump (case I)

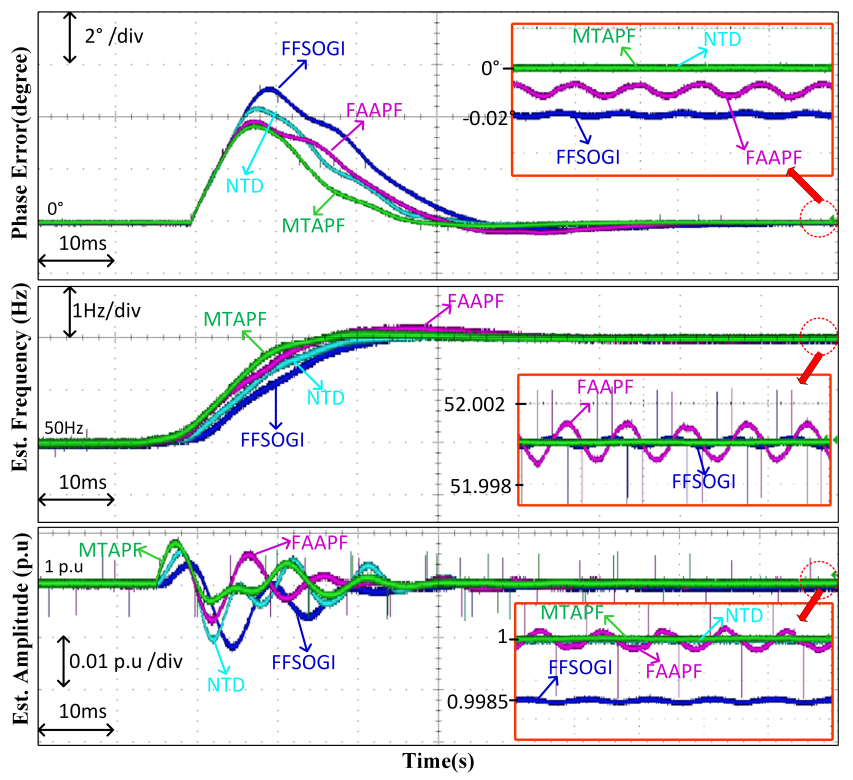

Fig. 15: Dynamic and SS performance in event of frequency change (case II)

The experimental evaluation of the dc offset and harmonic rejection capability of these PLL algorithms are demonstrated in Fig.17. A dc offset of 0.02 p.u is included in the input signal for test case IV and the estimated grid quantities are shown in Fig. 17 (a). All four demonstrate a similar level of dc offset rejection capability, with FAAPF having slightly lower peakto-peak oscillations. Similarly, for test case V, the grid voltage signal is polluted with $3^{r d}, 5^{t h}$ and $7^{t h}$ harmonics each with

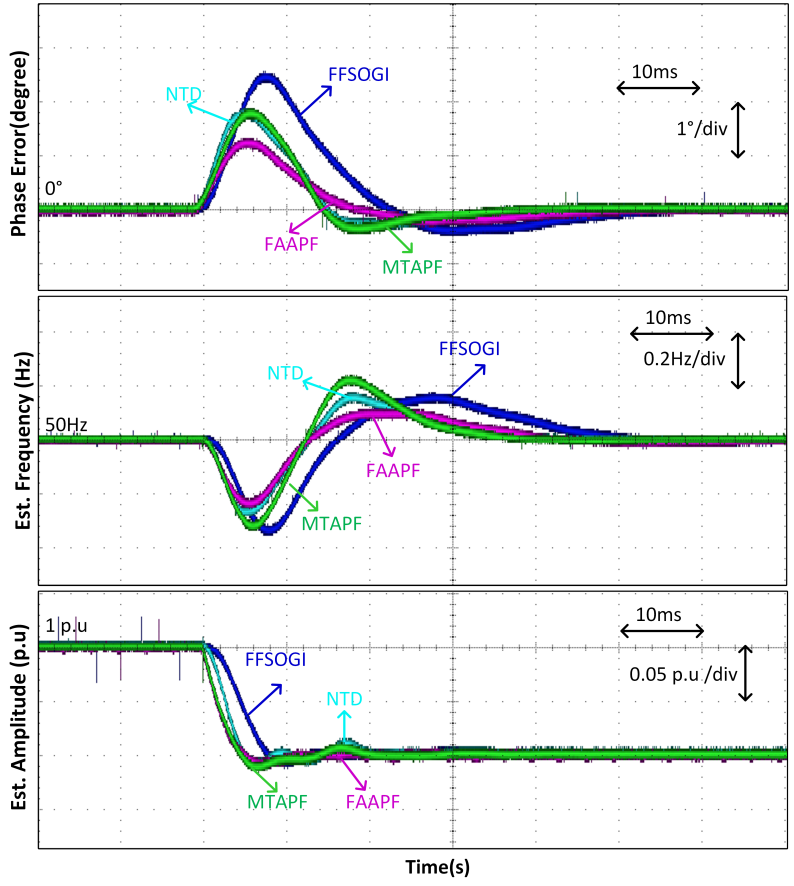

Fig. 16: Dynamic performance in event of amplitude jump (case III)

0.05, 0.04 and 0.03 p.u resulting in THD at $7 \%$ (EN50160 standard allows $8 \%$ [23]). The corresponding phase error, estimated frequency, and amplitude are sketched in Fig.17(b). The estimated grid parameters from the FFSOGI are more filtered with lower fluctuation levels. FAAPF and MTAPF both show higher amount of peak-to-peak oscillation in amplitude estimation. Unlike SOGI, the harmonic component output from APF are not attenuated but passed with unity gain while calculating $v_{d}$ component.

Case VI evaluates the responses of PLLs in grid frequency drift $(-2 \mathrm{~Hz})$ in the presence of harmonic distortion (with THD 7.3\%) and illustrated in Fig. 18. All four PLLs are able to track the grid quantities albeit different oscillatory errors. As expected FFSOGI has better harmonic rejection ability, while MTAPF and FAAPF display equivalent response with higher oscillations. However, MTAPF boasts of lower average phase error because of symmetricity around $0^{\circ}$.

Finally, the execution times of these four PLL algorithms in dSPACE platform are portrayed in Fig. 19 to evaluate their implementation complexity. MTAPF has marginally higher $(0.3 \mu s)$ processing time than FAAPF-PLL and lower to the rest of the methods. The results indicate MTAPF is computationally competitive with FAAPF and other FF-PLLs.

\section{CONCLUSION}

This paper explored four approaches to achieve the frequency fixed APF-PLL. A comprehensive comparison among them is conducted considering four predefined performance indices. The best among these (MTAPF) was then compared with the FAAPF-PLL and two other frequency-fixed PLLs: FFSOGI and NTD. The experimental results demonstrated that the MTAPF yields faster transient response than FFSOGI and 


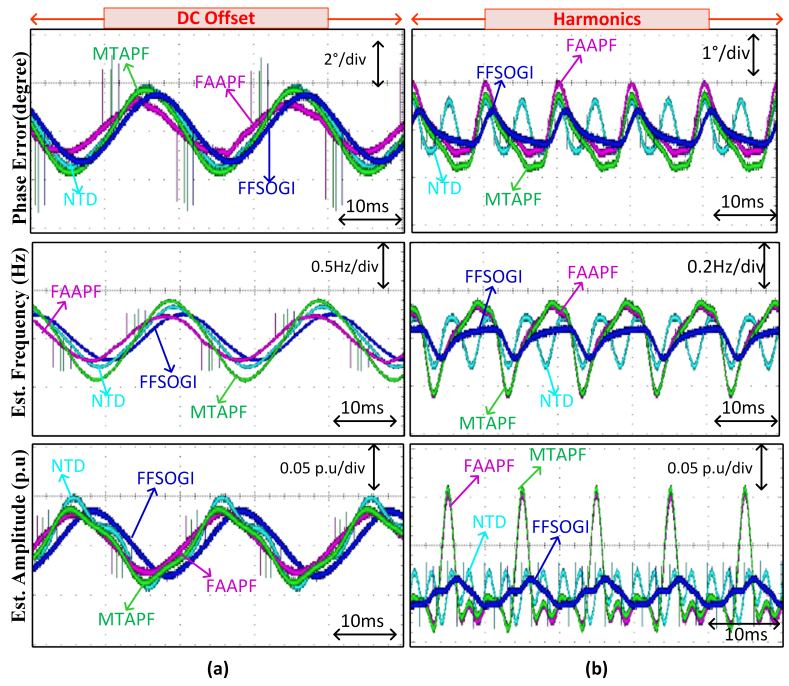

Fig. 17: Steady state performance of estimated quantities for (a) case IV (b) case V

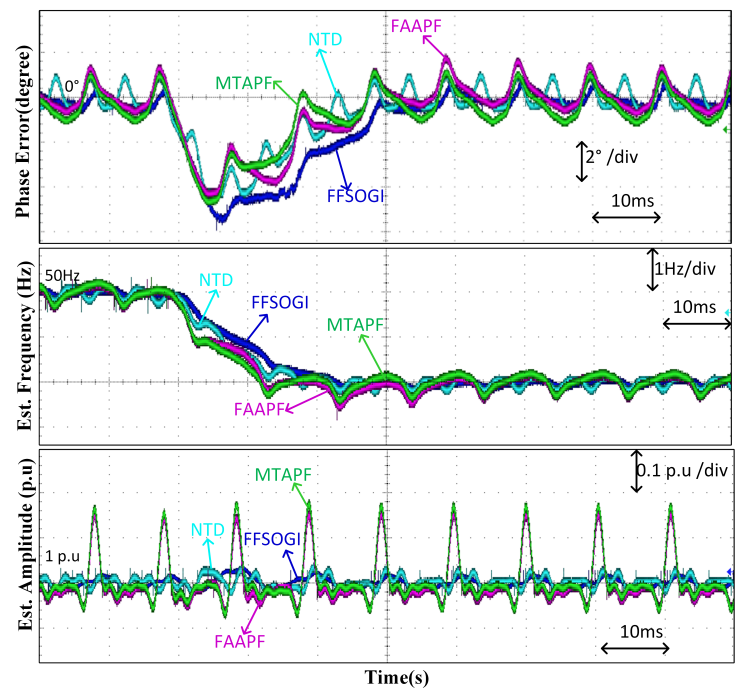

Fig. 18: Experimental result of PLL responses for case VI

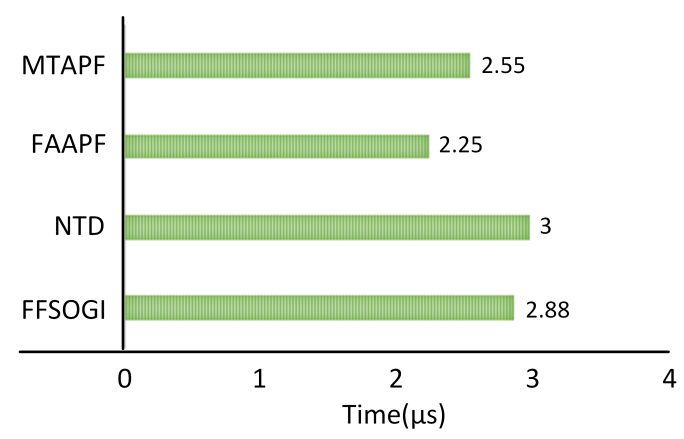

Fig. 19: Execution times of PLL algorithms in dSPACE

FAAPF, and comparable transient time with NTD-PLL, while also maintaining high accuracy in the estimated quantities. The solution based on the FAAPF has the most straightforward digital implementation, although it is complicated in the control system perspective. The MTAPF achieves the FF feature with only $13 \%$ increase in computational load with respect to FAAPF. The dc offset and harmonics rejection capability of MTAPF and FAAPF are almost identical with minor differences quantitatively. Integrating harmonics and dc offset rejection capability in MTAPF, will be the focus of our future work.

\section{REFERENCES}

[1] S. Ouchen, M. Benbouzid, F. Blaabjerg, A. Betka, and H. Steinhart, "Direct power control of shunt active power filter using space vector modulation based on super twisting sliding mode control," IEEE Journal of Emerging and Selected Topics in Power Electronics, pp. 1-1, 2020.

[2] A. Rahoui, A. Bechouche, H. Seddiki, and D. O. Abdeslam, "Virtual flux estimation for sensorless predictive control of pwm rectifiers under unbalanced and distorted grid conditions," IEEE Journal of Emerging and Selected Topics in Power Electronics, pp. 1-1, 2020.

[3] S. Biricik, H. Komurcugil, H. Ahmed, and E. Babaei, "Super twisting sliding mode control of dvr with frequency-adaptive brockett oscillator," IEEE Transactions on Industrial Electronics, pp. 1-1, 2020.

[4] A. Nicastri and A. Nagliero, "Comparison and evaluation of the pll techniques for the design of the grid-connected inverter systems," in 2010 IEEE International Symposium on Industrial Electronics, pp. 38653870, IEEE, 2010.

[5] S. Golestan, J. M. Guerrero, and J. C. Vasquez, "Single-phase plls: A review of recent advances," IEEE Transactions on Power Electronics, vol. 32, no. 12, pp. 9013-9030, 2017.

[6] S. Golestan, J. M. Guerrero, A. Vidal, A. G. Yepes, J. Doval-Gandoy, and F. D. Freijedo, "Small-signal modeling, stability analysis and design optimization of single-phase delay-based plls," IEEE Transactions on Power Electronics, vol. 31, no. 5, pp. 3517-3527, 2015.

[7] C. Zhang, O. Fosso, M. Molinas, et al., "Frequency domain modelling for assessment of hilbert and sogi based single-phase synchronisation," in IECON 2019-45th Annual Conference of the IEEE Industrial Electronics Society, vol. 1, pp. 1780-1785, IEEE, 2019.

[8] C. Subramanian and R. Kanagaraj, "Single-phase grid voltage attributes tracking for the control of grid power converters," IEEE Journal of Emerging and Selected Topics in Power Electronics, vol. 2, no. 4, pp. 1041-1048, 2014.

[9] A. Kherbachi, A. Chouder, A. Bendib, K. Kara, and S. Barkat, "Enhanced structure of second-order generalized integrator frequencylocked loop suitable for dc-offset rejection in single-phase systems," Electric Power Systems Research, vol. 170, pp. 348-357, 2019.

[10] S. Golestan, J. M. Guerrero, J. C. Vasquez, A. M. Abusorrah, and Y. AlTurki, "All-pass-filter-based pll systems: Linear modeling, analysis, and comparative evaluation," IEEE Transactions on Power Electronics, vol. 35, no. 4, pp. 3558-3572, 2019.

[11] A. Ohori, N. Hattori, and T. Funaki, "Phase-locked loop using complexcoefficient filters for grid-connected inverter," Electrical Engineering in Japan, vol. 189, no. 4, pp. 52-60, 2014.

[12] B. E. Luna, C. B. Jacobina, and A. C. Oliveira, "A new pll structure for single-phase grid-connected systems," in IECON 2011-37th Annual Conference of the IEEE Industrial Electronics Society, pp. 1081-1084, IEEE, 2011.

[13] H. Sagha, G. Ledwich, A. Ghosh, and G. Nourbakhsh, "A frequency adaptive single-phase phase-locked loop with harmonic rejection," in IECON 2014-40th Annual Conference of the IEEE Industrial Electronics Society, pp. 1028-1033, IEEE, 2014.

[14] T. Xia, X. Zhang, G. Tan, and Y. Liu, "All-pass-filter-based pll for singlephase grid-connected converters under distorted grid conditions," IEEE Access, vol. 8, pp. 106226-106233, 2020.

[15] S. Gautam, P. Yunqing, M. Kashif, Y. R. Kafle, Z. Z. Hua, and L. Bo, "Study on software phase locked loop for single phase active power filter," 2013.

[16] D. Stojić, N. Georgijević, M. Rivera, and S. Milić, "Novel orthogonal signal generator for single phase pll applications," IET Power Electronics, vol. 11, no. 3, pp. 427-433, 2017.

[17] A. Kulkarni and V. John, "A novel design method for sogi-pll for minimum settling time and low unit vector distortion," in IECON 2013 39th Annual Conference of the IEEE Industrial Electronics Society, pp. 274-279, IEEE, 2013. 
[18] M. Rasheduzzaman, S. Khorbotly, and J. W. Kimball, "A modified srfpll for phase and frequency measurement of single-phase systems," in 2016 IEEE Energy Conversion Congress and Exposition (ECCE), pp. 17, IEEE, 2016.

[19] A. Verma, R. Jarial, and U. M. Rao, "Modified non-adaptive bandpass filter based phase locked loop," Electronics Letters, vol. 56, no. 18, pp. 951-954, 2020.

[20] F. Xiao, L. Dong, L. Li, and X. Liao, "A frequency-fixed sogi-based pll for single-phase grid-connected converters," IEEE Transactions on Power Electronics, vol. 32, no. 3, pp. 1713-1719, 2016.

[21] A. Kulkarni and V. John, "Design of a fast response time single-phase pll with dc offset rejection capability," Electric Power Systems Research, vol. 145, pp. 35-43, 2017.

[22] S. Golestan, S. Y. Mousazadeh, J. M. Guerrero, and J. C. Vasquez, "A critical examination of frequency-fixed second-order generalized integrator-based phase-locked loops," IEEE Transactions on Power Electronics, vol. 32, no. 9, pp. 6666-6672, 2017.

[23] Y. Han, M. Luo, X. Zhao, J. M. Guerrero, and L. Xu, "Comparative performance evaluation of orthogonal-signal-generators-based singlephase pll algorithmsa survey," IEEE Transactions on Power Electronics, vol. 31, no. 5, pp. 3932-3944, 2015.

[24] T. Xia, X. Zhang, G. Tan, and Y. Liu, "Synchronous reference frame single-phase phase-locked loop (pll) algorithm based on half-cycle dft," IET Power Electronics, vol. 13, no. 9, pp. 1893-1900, 2020.

[25] P. Lamo, F. Lopez, A. Pigazo, and F. J. Azcondo, "An efficient fpga implementation of a quadrature signal-generation subsystem in srf plls in single-phase pfcs," IEEE Transactions on Power Electronics, vol. 32, no. 5, pp. 3959-3969, 2016.

[26] M. Ramezani, S. Golestan, and S. Li, "Non-frequency sensitive allpass filter based single-phase plls," in 2016 IEEE/PES Transmission and Distribution Conference and Exposition (T\&D), pp. 1-5, IEEE, 2016.

[27] U.-S. Seong and S.-H. Hwang, "Analysis of phase error effects due to grid frequency variation of srf-pll based on apf," Journal of Power Electronics, vol. 16, no. 1, pp. 18-26, 2016.

[28] B. Liu, M. An, H. Wang, Y. Chen, Z. Zhang, C. Xu, S. Song, and Z. Lv, "A simple approach to reject dc offset for single-phase synchronous reference frame pll in grid-tied converters," IEEE Access, vol. 8, pp. 112297-112308, 2020.

[29] H. Sagha, Development of innovative robust stability enhancement algorithms for distribution systems containing distributed generators. $\mathrm{PhD}$ thesis, Queensland University of Technology, 2015.

[30] H. Khazraj, F. F. da Silva, C. L. Bak, and S. Golestan, "Analysis and design of notch filter-based plls for grid-connected applications," Electric Power Systems Research, vol. 147, pp. 62-69, 2017.

[31] S. Golestan, M. Monfared, F. D. Freijedo, and J. M. Guerrero, "Design and tuning of a modified power-based pll for single-phase grid-connected power conditioning systems," IEEE Transactions on Power Electronics, vol. 27, no. 8, pp. 3639-3650, 2012.

[32] J. Kaiser and R. Hamming, "Sharpening the response of a symmetric nonrecursive filter by multiple use of the same filter," IEEE Transactions on Acoustics, Speech, and Signal Processing, vol. 25, no. 5, pp. 415422, 1977.

[33] S. Gautam, Y. Lu, W. Xiao, D. D.-C. Lu, and M. S. Golsorkhi, "Dualloop control of transfer delay based pll for fast dynamics in single-phase ac power systems," IET Power Electronics, vol. 12, no. 13, pp. 35713581, 2019. 